\title{
ELSEVIER_YADPA_51119
}

\section{Signet-ring cell lymphoma: clinicopathologic, immunohistochemical, and fluorescence in situ hybridization studies of 7 cases}

Shanxiang Zhang, MD, PhD ${ }^{a, *}$

sz5@iupui.edu

Jihong Sun, MDa

Yanan Fang, MD

Mehdi Nassiri, MDa

Lanting Liu, MD

Jiehao Zhou, MD, PhDa

Ryan Stohler, BS

Haki Choi, MS

Gail H Vance, MDa,d

aDepartment of Pathology \& Laboratory Medicine, Indiana University School of Medicine, Indianapolis, IN

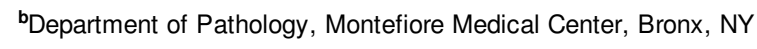

cAmeriPath, Indianapolis, IN

${ }^{\mathrm{d} D e p a r t m e n t ~ o f ~ M e d i c a l ~ a n d ~ M o l e c u l a r ~ G e n e t i c s, ~ I n d i a n a ~ U n i v e r s i t y ~ S c h o o l ~ o f ~ M e d i c i n e, ~ I n d i a n a p o l i s, ~ I N ~}$

*Corresponding author at: Department of Pathology and Laboratory Medicine, Indiana University, 350 West 11th Street, Room 5042, Indianapolis, IN 46202. Tel.: +1 3174916175 ; fax: +1 317 4916114.

Keywords: Signet-ring cell lymphoma; Immunohistochemical; Fluorescence in situ hybridization

\section{Introduction}

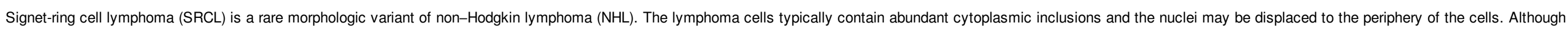

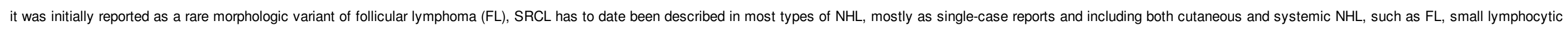

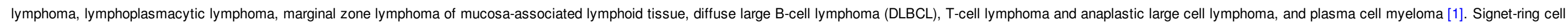

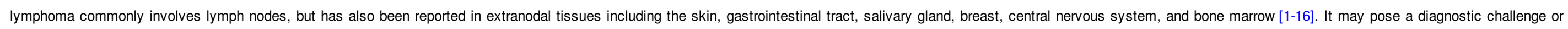
even be misdiagnosed, especially in the absence of an extensive immunohistochemical (IHC) study and flow cytometric analysis due to a limited needle biopsy sample [6,17].

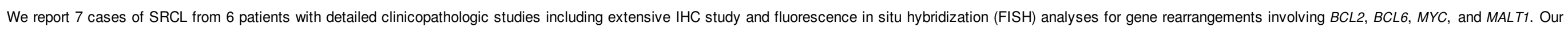
study demonstrated that an extensive IHC study is preferred for adequate diagnosis and classification of SRCL.

\section{Materials and methods}

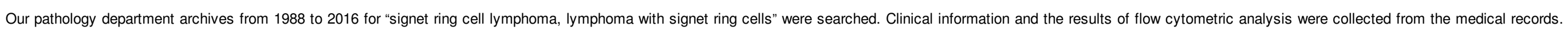
Five cases from 4 patients were identified. Two additional cases were contributed by the collaborating colleagues. This study was approved by the institutional review board of our institute.

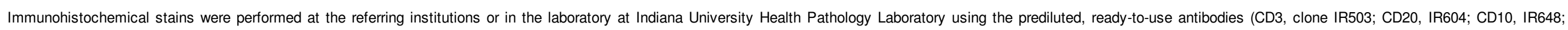
BCL2, IR614; BCL6, IR625; Ki-67, IR626; MUM-1, IR644) from Dako with a Dako Autostainer Plus instrument following the manufacturer's protocols [18].

This is the author's manuscript of the article published in final edited form as:

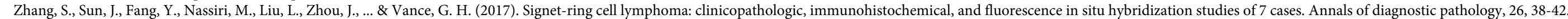
https://doi.org/10.1016/j.anndiagpath.2016.10.002 


\section{ELSEVIER_YADPA_51119}

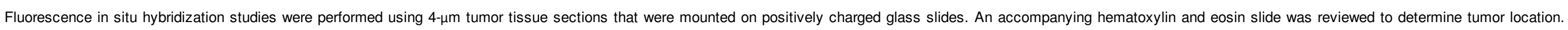

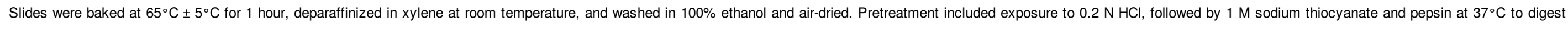

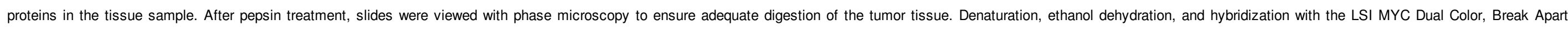

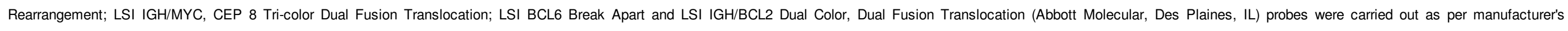

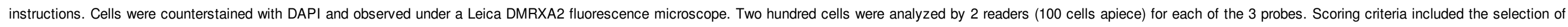
single nuclei with representation of at least 1 signal for each color/probe/nucleus.

\section{Results}

\subsection{Clinical features}

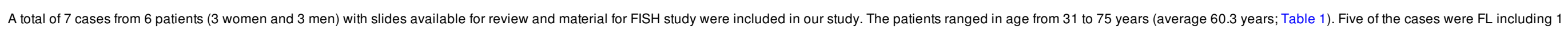

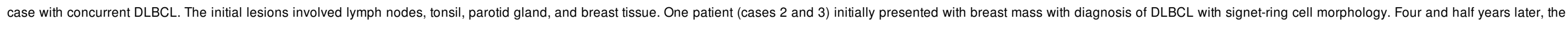
patient developed extensive lymphadenopathy and a thigh mass which was subsequently diagnosed as low-grade FL with signet-ring cell morphology.

Table 1 Summary of clinicopathologic features and follow-up

\section{alt-text: Table 1}

\begin{tabular}{|c|c|c|c|c|c|c|c|}
\hline Case & Age $(y)$ & Sex & Site of biopsy & Stage & Diagnosis & Treatment & Follow-up \\
\hline 1 & 31 & $\mathrm{~F}$ & Parotid, LN & II & DLBCL, FL 3 & $\mathrm{R}-\mathrm{CHOP} \times 6$ & Local recurrence at $20 \mathrm{mo}$ \\
\hline 2 & 71 & $\mathrm{~F}$ & Breast & III & DLBCL & Chemotherapy $\times 8$ & Dead 4.5 y \\
\hline 3 & 75 & $\mathrm{~F}$ & Thigh mass & Unknown & FL, low-grade & Unknown & Dead, 4 mo later \\
\hline 4 & 60 & M & Submandibular LN & Unknown & FL, $1-2$ & Resection + radiation & Free of disease \\
\hline 5 & 60 & M & $\mathrm{LN}$ & Unknown & FL 3 & Unknown & Not available \\
\hline 6 & 52 & $\mathrm{~F}$ & Tonsil & Unknown & DLBCL, MZL & Unknown & Not available \\
\hline 7 & 73 & M & $\mathrm{LN}$ & IIIA & FL, $1-2$ & Observation & Stable \\
\hline
\end{tabular}

Abbreviations: LN, lymph node; MZL, marginal zone lymphoma; R-CHOP, cyclophosphamide, doxorubicin, vincristine, prednisone, and rituximab.

\subsection{Histopathologic features}

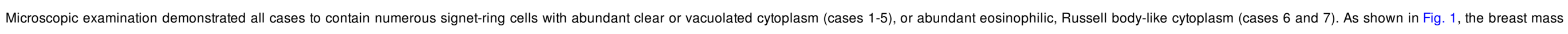

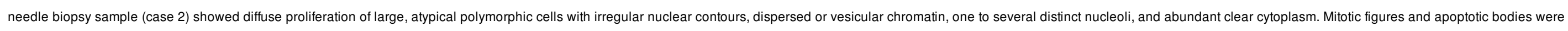

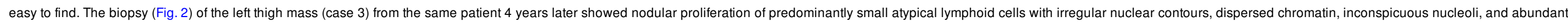

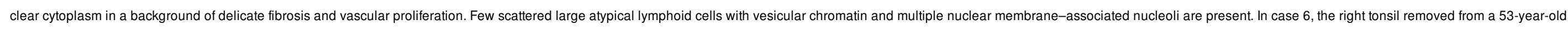

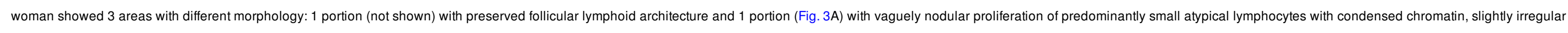

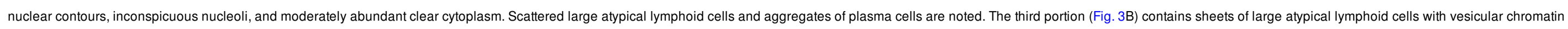

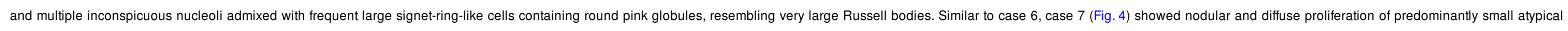
lymphoid cells with abundant eosinophilic cytoplasm pushing the nuclei to the periphery of cells. 


\section{ELSEVIER_YADPA_51119}

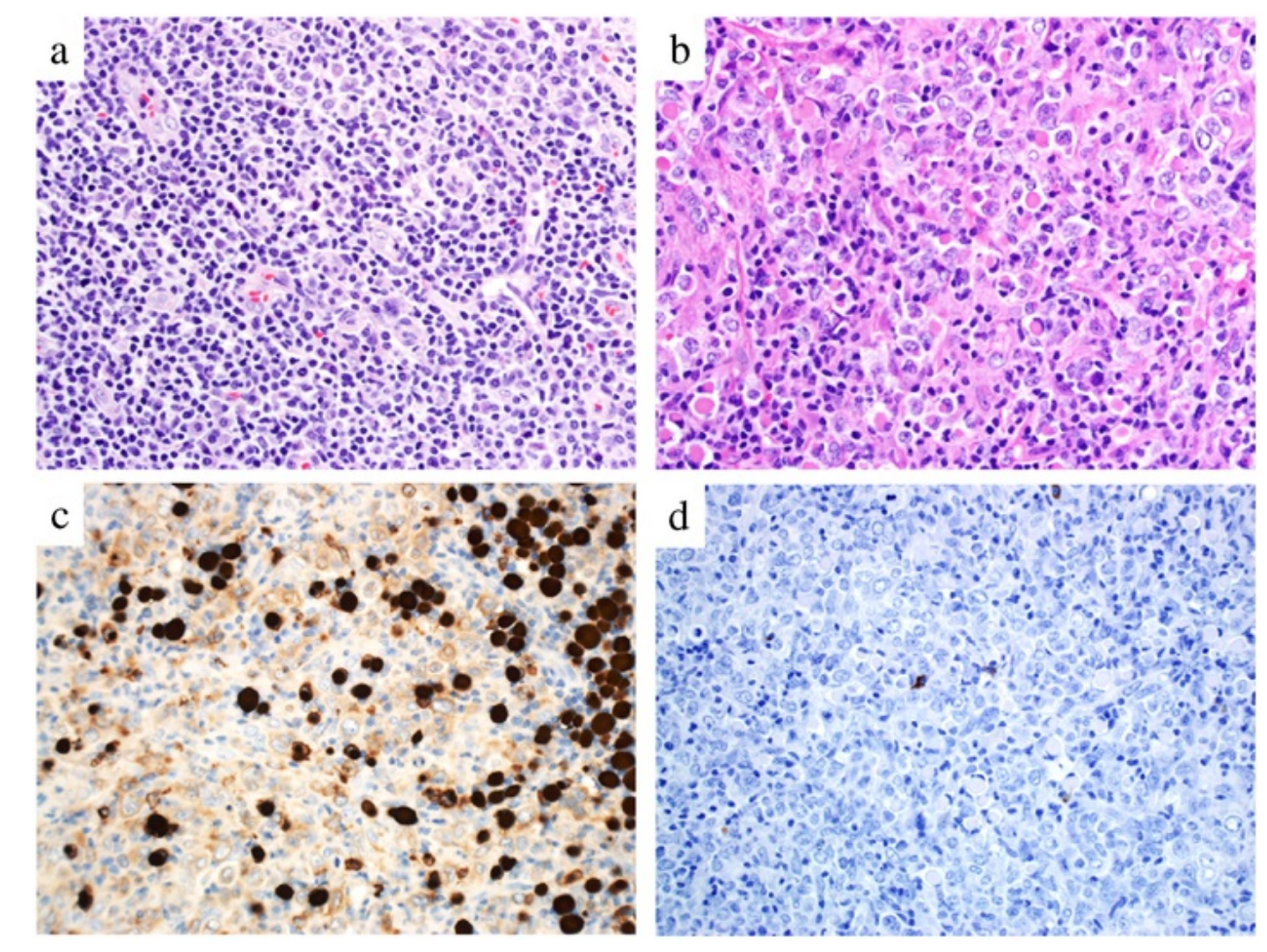

Fig. 3 (A) Low-grade lymphoma (case 6; hematoxylin and eosin, original magnification $\times 50$ ). (B-D) Large B-cell lymphoma with signet-ring morphology (hematoxylin and eosin, $\times 50$ [B]; immunohistochemistry, $\times 40$ [C-D]). $\mathrm{K}(\mathrm{F})$ and $\lambda$ (D). alt-text: Fig. 3 


\section{ELSEVIER_YADPA_51119}
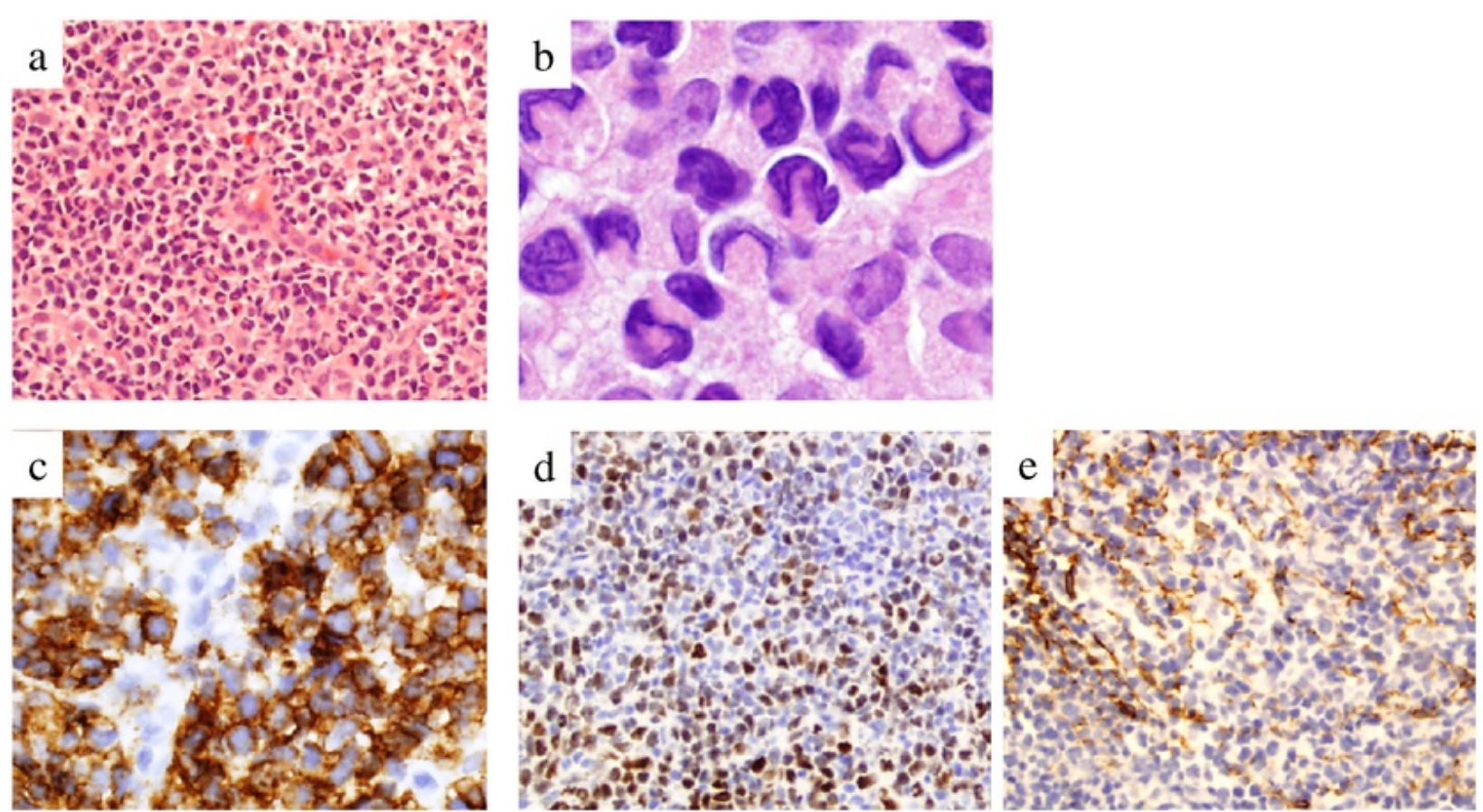

Fig. 4 Follicular lymphoma with signet-ring morphology (case 7). (A and B) Hematoxylin and eosin, original magnifications $\times 20$ (A) and ×100 (B). (C-E) Immunohistochemistry, $\times 20$ : CD20 (C), BCL-6 (D), and CD21 (E), alt-text: Fig. 4

\subsection{Immunohistochemical findings}

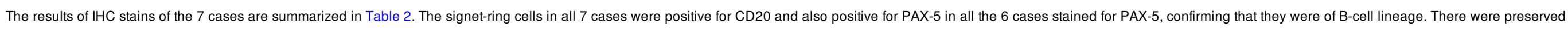

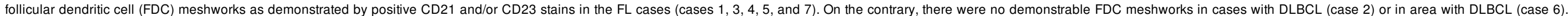

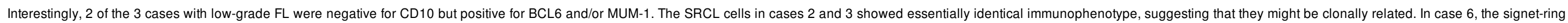

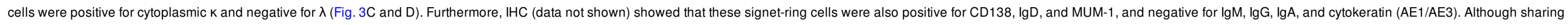

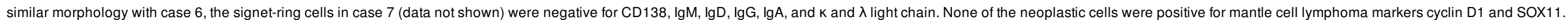

Table 2 Summary of IHC stains

\begin{tabular}{|c|c|c|c|c|c|c|c|c|c|c|c|c|c|}
\hline Case & CD3 & CD5 & CD20 & PAX5 & $\mathrm{CD} 23$ & CD10 & BCL2 & BCL6 & MUM1 & CD21 & Cyclin D1 & SOX11 & Ki-67 \\
\hline 1 & - & - & + & + &,$+{ }^{*}$ & + & + & + & - & * & - & - & $70 \%$ \\
\hline 2 & - & - & + & + & - & - & + & $+/-$ & + & ${ }^{*}$, focal & - & - & $10 \%-40 \%$ \\
\hline 3 & - & - & + & + & - & - & + & $+/-$ & $+/-$ & * & - & - & $20 \%$ \\
\hline 4 & - & - & + & + &,$-{ }^{*}$ & - & + & + & - & - & - & - & $20 \%-30 \%$ \\
\hline 5 & - & - & + & + &,$-{ }^{*}$ & + & + & - & - & - & - & - & $20 \%$ \\
\hline 6 & - & ND & + & + & ND & - & + & + & + & ${ }^{*}$, focal & - & ND & $30 \%-40 \%$ \\
\hline
\end{tabular}




\section{ELSEVIER_YADPA_51119}

\begin{tabular}{l|l|l|l|l|l|l|l|}
7 & ND
\end{tabular}

Abbreviations: *, positive for FDC meshwork; ND, not done.

\subsection{Fluorescence in situ hybridization findings}

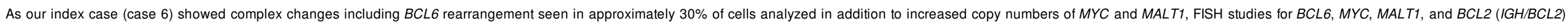

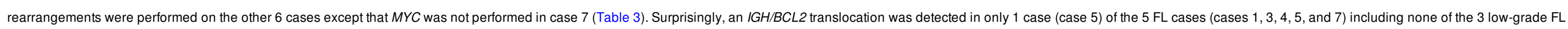
cases (cases 3,4 , and 7). There was also increased MALT1 copy number in case 7 . Case 3 showed copy number changes for both $B C L 6$ and MYC. No abnormalities were detected in the remainder of the cases.

\section{Table 3 Summary of FISH study}

\begin{tabular}{|c|c|c|c|c|c|}
\hline Case & IGH/BCL2 & BCL6 & MYC & MALT1 & API2/MALT1 \\
\hline 1 & - & - & - & - & ND \\
\hline 2 & - & - & - & - & ND \\
\hline 3 & - & 3 copies & 1 copy & - & - \\
\hline 4 & - & - & - & - & ND \\
\hline 5 & + & - & - & - & ND \\
\hline 6 & - & $30 \% \mathrm{R}$ & 3-7 copies & 3-6 copies & ND \\
\hline 7 & - & - & ND & 3-4 copies & - \\
\hline
\end{tabular}

Abbreviations: ND, not done; R, rearranged.

\subsection{Clinical follow-up}

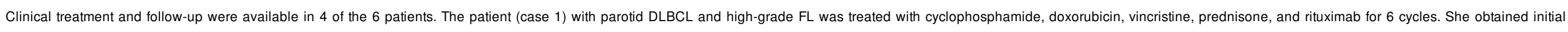

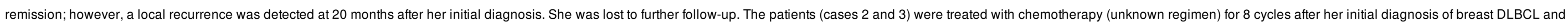

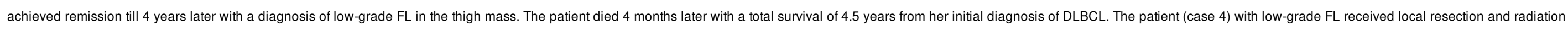

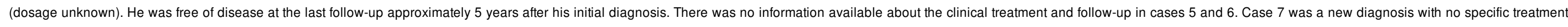
currently.

\section{Discussion}

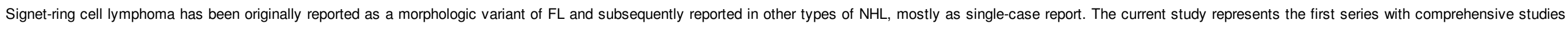

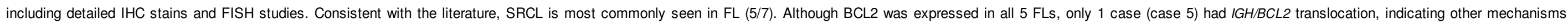

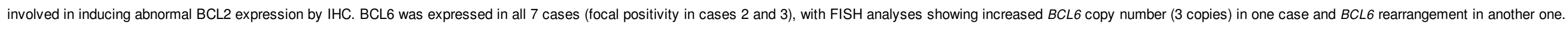

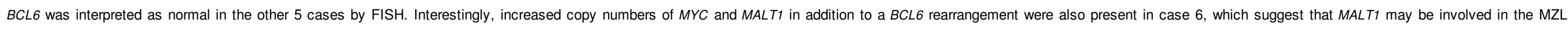

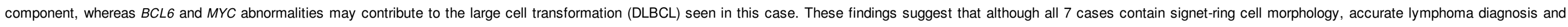
classification require detailed IHC study.

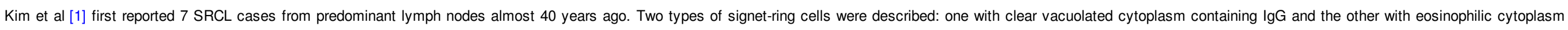

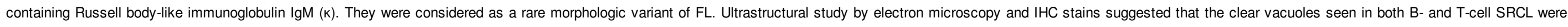




\section{ELSEVIER_YADPA_51119}

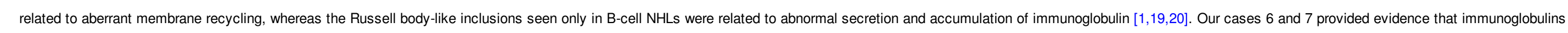
other than IgM ( $\kappa$; case 6) or other nonimmunoglobulin proteins (case 7) may also accumulate inside the cytoplasm, contributing to the prominent signet-ring cell morphology.

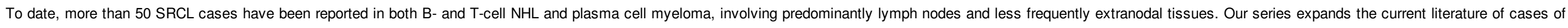

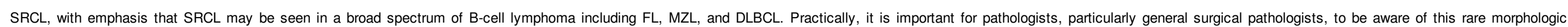

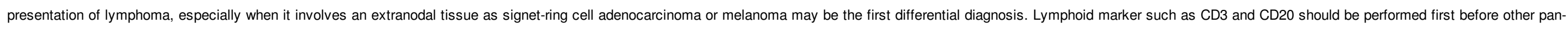

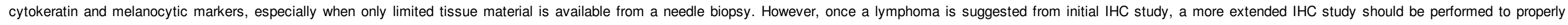
classify the lymphoma according to current World Health Organization classification.

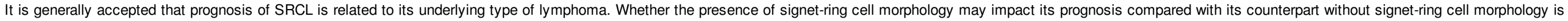

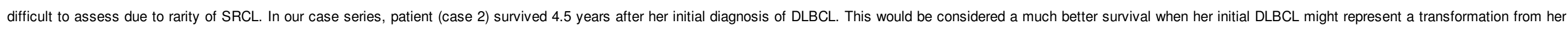

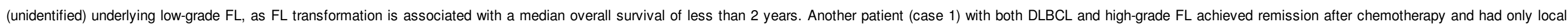
recurrence at 20 months after initial diagnosis.

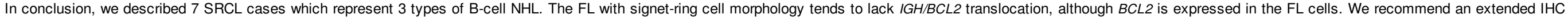
study to include minimally CD3, CD20, CD5, CD10, CD21, CD23, BCL2, BCL6, and cyclin D1 for correct diagnosis and classification of SRCL.

\section{References}

[1]

H. Kim, R.F. Dorfman and H. Rappaport, Signet ring cell lymphoma. A rare morphologic and functional expression of nodular (follicular) lymphoma, Am J Surg Pathol 2 (2), $1978,119-132$.

\section{[2]}

P.A. Allevato, et al., Signet ring cell lymphoma of the thyroid: a case report, Hum Pathol 16 (10), 1985, 1066-1068.

\section{[3]}

F. Baranyay, et al., Signet-ring cell lymphoma, Morphol lgazsagugyi Orv Sz 22 (1), 1982, 27-32.

\section{[4]}

N. Basir, et al., Signet ring cell lymphoma of the small bowel: a case report, Oman Med J 27 (6), 2012, 491-493.

[5]

C. Bellas, et al., Signet-ring cell lymphoma of T-cell type with CD30 expression, Histopathology 22 (2), 1993, 188-189.

[6]

A.M. Bogusz, et al., Extreme signet ring cell change in a large B-cell lymphoma of follicular origin, Int J Surg Pathol 21 (4), 2013, 399-403.

\section{[7]}

B.N. Coffing and M.S. Lim, Signet ring cell lymphoma in a patient with elevated CA-125, J Clin Oncol Off J Am Soc Clin Oncol 29 (14), 2011, e416-e418.

[8]

P.A. Cross, B.P. Eyden and M. Harris, Signet ring cell lymphoma of T cell type, J Clin Pathol 42 (3), 1989, 239-245.

[9]

P.J. Dolman, J. Rootman and N.F. Quenville, Signet-ring cell lymphoma in the orbit: a case report and review, Can J Ophthalmol 21 (6), 1986, $242-245$. 


\section{ELSEVIER_YADPA_51119}

B. Falini, et al., CD30+ anaplastic large-cell lymphoma, null type, with signet-ring appearance, Histopathology 30 (1), 1997, 90-92.

\section{[11]}

M. Harris, B. Eyden and G. Read, Signet ring cell lymphoma: a rare variant of follicular lymphoma, J Clin Patho/ 34 (8), 1981, 884-891.

\section{[12]}

W.G. McCluggage, et al., B cell signet-ring cell lymphoma of bone marrow, J Clin Pathol 48 (3), 1995, 275-278.

[13]

C.A. Moran, S. Suster and S.L. Abbondanzo, Cutaneous B-cell lymphoma with signet ring-cell morphology: a clinicopathologic and immunohistochemical study of three cases, Am J Dermatopathol 23 (3), 2001, 181-184.

[14]

D. Ramnani, et al., Signet-ring cell variant of small lymphocytic lymphoma with a prominent sinusoidal pattern, Ann Diagn Pathol 3 (4), 1999, $220-226$.

[15]

S.C. van der Putte, et al., T-cell signet-ring cell proliferation in the skin simulating true histiocytic lymphoma, Am J Dermatopathol 9 (2), 1987, 120-128.

[16]

L.M. Weiss, G.S. Wood and R.F. Dorfman, T-cell signet-ring cell lymphoma. A histologic, ultrastructural, and immunohistochemical study of two cases, Am J Surg Patho/ 9 (4), 1985, 273-280.

$[17]$

J.R. Krause, Signet ring lymphoma: a potential diagnostic mishap, Proceedings 26 (3), 2013, 293-294.

[18]

Dako, www.dako.com.

[19]

J.G. van den Tweel, et al., Immunoglobulin inclusions in non-Hodgkin's lymphomas, Am J Clin Pathol 69 (3), 1978, 306-313.

\section{[20]}

J.J. Navas-Palacios, M.D. Valdes and J.J. Lahuerta-Palacios, Signet-ring cell lymphoma. Ultrastructural and immunohistochemical features of three varieties, Cancer 52 (9), $1983,1613-1623$.

\section{Queries and Answers}

Query:

The author names have been tagged as given names and surnames (surnames are highlighted in teal color). Please confirm if they have been identified correctly.

\section{Answer: Yes}

Query:

Keywords were provided. Please check if appropriate.

Answer: Please see the attached new keywords.

Query:

Please provide an abstract.

Answer: Please see attached. 


\section{ELSEVIER_YADPA_51119}

\section{Query:}

Please provide manufacturer's city and state/country.

Answer: Santa Clara, CA /USA

Query:

Please list the names of first 6 authors before using "et al."

Answer: Please see the attached. 